\title{
Un nuevo sujeto a las puertas de la política social pública
}

\section{Mabel Cenizo}

Caritas Gipuzkoa

<formazioa@caritasgi.org>

\section{Fernando Echarte}

Caritas Gipuzkoa

\section{Jon Telletxea}

Caritas Gipuzkoa
Gure gizartean dogma gisara ezarri diren ziurtasun sozial batzuek - 'lan eginez gero, ez zara pobre izango'; 'ikasiz gero, lan egoki bat izango duzu'; edo 'betiere hobetuko duzu eta aurrerabidea ezagutuko duzu'- atzera bueltako aldaketa ezagutzen ari dira, eta lardaskatu ditu kohesio, gizarteratze eta parte-hartze sozialeko prozesuak. Eraldaketa hau gertatzen da krisi sozioekonomiko baten garaian, eta testuinguru honetan enpleguak galdu egin du bere izaera inklusiboa, hobekuntza makroekonomikoak ez dira heltzen pobre izaten jarraitzen duten etxebizitzetara, eta hazi egiten da ziurgabetasunaren sentsazioa eta arriskuaren hautematea. Artikulu honen xedea da, egungo testuinguru sozial eta ekonomikotik, bere burua bazterkerian hauteman ez arren, integratua sentitzen ez den kaltebera den subjektu baten emergentzia eta, besteak beste, ez ditu zalantzan jartzen bere egoera epe ertain edo luze batean hobetzeko babes sozialeko sistema, gizarteratzeko estrategia eta enplegu-politiken gaitasuna.

\section{GAKO-HITZAK:}

Gizarte-bazterketa, ezegonkortasuna, arriskua, langabezia, gizarte-politika, hirugarren sektorea.
Algunas certezas sociales -'si trabajas, no eres pobre'; 'si estudias, tendrás un buen trabajo'; o 'siempre irás a mejor y progresarás'- que se han impuesto en nuestra sociedad como dogmas están sufriendo cambios irreversibles que están trastocando los procesos de cohesión, inclusión y participación social. Esta transformación se produce en medio de una crisis socioeconómica donde parte del empleo ha perdido su propiedad inclusiva, las mejoras macroeconómicas no llegan a los hogares que siguen siendo pobres, y crece la sensación de incertidumbre y la percepción de amenaza. El objetivo de este artículo es explicar, desde el contexto social y económico actual, la emergencia de un sujeto vulnerable que no se percibe excluido, pero que tampoco se siente integrado y que nos cuestiona, entre otros, el sistema de protección social, las estrategias de inclusión, y las políticas de empleo, que no le permiten mejorar su situación ni a medio ni a largo plazo.

\section{Palabras ClaVe:}

Exclusión social, precariedad, riesgo, desempleo, política social, tercer sector. 


\section{Introducción}

Como decía Ulrick Beck (2017: 19), “el mundo está experimentando una sorprendente pero comprensible metamorfosis mediante la transformación del horizonte referencial”. Algunas certezas sociales - 'si trabajas, no eres pobre'; 'si estudias, tendrás un buen trabajo’; o ‘siempre irás a mejor y progresarás'- que se han impuesto en nuestra sociedad como dogmas están sufriendo cambios irreversibles que están trastocando los procesos de cohesión, inclusión y participación social. Esta transformación se produce en un contexto de crisis socioeconómica, donde parte del empleo ha perdido su propiedad inclusiva, las mejoras macroeconómicas no llegan a los hogares que siguen siendo pobres, y crece en la población en general la sensación de incertidumbre y la percepción de amenaza.

Cada día, más personas de nuestros entornos laborales y vitales se perciben en una zona de inseguridad vital y precariedad laboral, y nos preguntan para cuestionarnos, como Caritas y tercer sector, qué hacemos con 'sus' situaciones de vulnerabilidad, cómo las atendemos y qué respuestas les ofrecemos. Ello abre un debate en el tercer sector y en la administración pública sobre las situaciones de vulnerabilidad, su demanda creciente de atención y su legitimidad para reclamar un lugar social, debate que nos obliga a buscar otras estrategias y otro marco conceptual de intervención social más acordes con estos perfiles de excusión social.

Éste es nuestro objetivo en este artículo: explicar, desde el contexto social y económico actual, la emergencia de un sujeto vulnerable que no se percibe excluido, pero que tampoco se siente integrado y que nos cuestiona, entre otros, el sistema de protección social, las estrategias de inclusión y las políticas de empleo, que no le permiten mejorar su situación ni a medio ni a largo plazo.

\section{Eso que nombramos como 'vulnerabilidad social'}

Para analizar más detenidamente esos riesgos de la vida que nos parecen inconexos, necesitamos desenfocar la mirada y ampliar la visión sobre cómo se articulan los diferentes grupos sociales en función de los recursos que tienen. A partir de esos recursos personales, sociales y económicos, podemos dibujar una gráfica con dos grandes ejes: el eje de la integración y el eje de la exclusión, y ubicar en ella a toda la sociedad.

Ese lugar social intermedio que define y transita entre los extremos de la integración y la exclusión social es una brecha tan amplia como indefinida, que abarca una pluralidad de grupos sociales que se encuentran en situaciones muy diversas y, sin embargo, comparten un espacio común: el espacio de la vulnerabilidad, también denominado precariedad, fragilidad o riesgo social. Un espacio poco medido, poco evaluado y poco presente en las políticas de protección social como concepto y foco de actuación, quizás, por la dificultad de acotar su volumen, de perfilar los grupos afectados y por la baja o media intensidad de indicadores que podrían dar lugar posteriormente a situaciones de exclusión social.

En la tipología de Robert Castel (cit. en Arriba, 2002), se distinguen tres niveles o zonas de integración social:

- Zona de integración, seguridad o estabilidad: corresponde a la situación típica-ideal de la población con trabajo, protección social asegurada y sólida relación familiar y vecinal.

- Zona de vulnerabilidad, precariedad o inestabilidad: se caracteriza por la fragilidad, la inseguridad de las relaciones laborales precarias y la inadecuación de los soportes familiares y sociales.

- Zona de exclusión o marginación: se caracteriza por la salida del mundo laboral, la ausencia de otro tipo de protección social y el aislamiento social. Quienes se encuentran aquí sufren las formas más extremas de pobreza, carecen de acceso a las formas normalizadas de participación social y son incapaces de salir por sí solos de esa situación.

Las tres zonas están determinadas, a su vez, por la posición de las personas en el mercado de trabajo (empleos estables y con buenas condiciones laborales frente a empleos precarios o falta de empleo), por la protección social asegurada y por las redes sociales de apoyo. Precisamente, el espacio de la vulnerabilidad social será el espacio de la precariedad laboral, el de la protección social no asegurada y el de la fragilidad relacional o social.

Nos parece interesante sumar a este análisis el eje 'sentido vital' que aporta Joaquín García Roca (1998), indicador que añade significaciones vitales a las personas. Ese recurso representa las fortalezas y capacidades personales y colectivas que permiten el vínculo, las relaciones, la capacidad de esperar, de luchar y de proyectarse en el futuro. Sin embargo, el contexto actual, donde crece la percepción de amenaza y la sensación de incertidumbre, no poder proyectarnos en el futuro a medio plazo, incluso teniendo un empleo o relaciones más o menos sólidas, constituye una ruptura con respecto a la sociedad que hemos conocido hasta fechas recientes.

Queremos centrarnos en el espacio de precariedad, un espacio donde habita un grupo de personas de nuestro entorno, personas jóvenes en busca de su primer empleo estable, o que no pueden acceder a una vida emancipada por la dificultad de acceso a una vivienda o un empleo precario, parejas jóvenes que posponen su descendencia, mujeres que encabezan familias monoparentales, personas 
migrantes, personas desempleadas de larga duración y trabajadoras pobres, entre otras. Queremos debatir sobre la emergencia de estas situaciones vitales que están a las puertas de nuestras instituciones sociales y que muestran, como puntas de iceberg, señales de emergencia que reclaman protección y lugar en los sistemas, en las intervenciones y en los planes de inclusión para mejorar sus condiciones de vida.

\section{Un contexto social precario y un horizonte conflictivo}

Tradicionalmente, uno de los espacios más integrados ha sido la vida laboral, construida sobre la base de certezas y 'perspectivas sociales' predeterminadas, que nos invitaban a seguir el sendero establecido para llegar al éxito personal, al progreso social y al desarrollo económico. A grandes rasgos, el itinerario vital y laboral de las últimas décadas estaba, más o menos, ordenado y enlazado, y era progresivo.

Al mismo tiempo, ese binomio vida-empleo se ha convertido en un imaginario social muy potente, institucionalizado en las cartas magnas y proyectado como meta y punto de partida para la construcción personal, familiar y social. En este sentido, el objetivo económico social principal de nuestras sociedades es recuperar el suficiente crecimiento económico para generar empleo.

Sin embargo, estos años de crisis global, por su intensidad y duración, han agravado rupturas sociales, que se han extendido por espacios sociales antes integrados; han desdibujado trayectorias y han dejado a los caminantes a su emprendedora suerte y a la intemperie institucional. Se ha producido una brecha en el subsuelo del empleo, que ha añadido más grietas a la realidad social - preguntemos, si no, a los jóvenes-. Esas grietas no son sólo económicas o laborales, si tenemos ingresos y empleo para gestionar la vida, sino que invisibilizan y van restando valor a todo lo que sucede fuera de ese marco concreto y cerrado del empleo, y por lo tanto, precarizan la vida y el cuidado de ésta, afectan a las decisiones que tomamos sobre emancipación y crianza, dificultan la conciliación y profundizan en la crisis de reproducción social que tienen nuestras envejecidas sociedades.

Esa precariedad en la esfera de la vida, que sufren fundamentalmente las mujeres, se instaura en los hogares, provocando, también, que los afectos, la seguridad psicológica y la creación de lazos humanos sean cada vez más difíciles de mantener para amplios colectivos sociales, que caen, vía relacional, en la zona de la vulnerabilidad (Carrasco, 2001). Ambas vulnerabilidades, las producidas por los riesgos laborales y las ocasionadas por los riesgos en los cuidados de la vida, están estrechamente relacionadas y refuerzan dinámicas endógenas de reproducción de la vulnerabilidad, tal como venía sucediendo en el espacio de la exclusión.
Muchas personas se sienten perdedoras del actual sistema económico, y desprotegidas también de un Estado del bienestar que no les asegura una existencia mínima frente a las incertidumbres y bamboleos del mercado laboral con el que se pelean. Sin esperarlo, desconcertadas, han quedado expuestas a los riesgos, a la incertidumbre y a su incansable suerte. No nos extraña que algunos movimientos sociales y ecos políticos se hayan erigido en defensa 'de lo nuestro' en el mundo occidental, y estén relacionados con este sentimiento angustioso y desesperanzador.

Este conflicto de supervivencia entre quienes están siendo precarizados o expulsados del mercado laboral (y no se sienten actualmente ni excluidos ni protegidos por el Estado) y quienes, también en situación de exclusión, sí están protegidos, de alguna manera, por la administración pública ejerce como vector de presión social hacia nuestro sistema social y democrático, rompiendo su principio de igualdad y solidaridad. Deducimos, que esta presión social está, entre otras, en el trasfondo del cuestionamiento del propio sistema de protección social, de los movimientos sociales y políticos que cierran puertas y fronteras a personas inmigrantes y refugiadas, del fenómeno del racismo y de la xenofobia, y de comentarios próximos cuando preguntamos ‘y de lo nuestro, ¿qué?’.

\section{La vulnerabilidad a nuestras puertas}

Por los argumentos anteriores, nos parece interesante poner el foco de atención en la vulnerabilidad y sus riesgos, además de acercarnos al posible volumen y perfil de personas que puedan ubicarse en la Comunidad Autónoma Vasca (CAV) en ese espacio social. Asimismo queremos preguntarnos, ante esta creciente realidad, si necesitamos otras políticas públicas, si debemos reforzar los sistemas de protección social, cambiar las estrategias de inclusión, repensar las herramientas a utilizar en los planes, para poner así en el centro la protección, la autonomía y la igualdad de oportunidades de todas las personas. Por supuesto, también de aquellas no consideradas en situación de exclusión y que, sin embargo, no están ni se proyectan en la 'zona de confort' de la integración plena.

Estas realidades sociales también nos deben hacer reflexionar, como Caritas y tercer sector, y preguntarnos cuál es nuestra visión y narrativa de estas situaciones de vulnerabilidad, cuál es nuestra misión ante ellas, si nos sentimos convocados para acompañarlas y contrarrestarlas, qué herramientas diferentes necesitamos articular, y cómo compaginar y complementar 'las luchas' y 'necesidades' de las personas en situación de exclusión con las de las personas en situación de vulnerabilidad, apelando a la carta de ciudadanía que nos ampara a todos y todas. Tenemos el reto de construir un imaginario posible, una narrativa dignificadora para las personas con empleo 0 
sin él, poniendo en valor otros elementos sociales que descartamos; valorando el aprendizaje, la formación para el empleo y también para la comunidad; ampliando la estrategia de la inclusión social hacia el mundo de los trabajos. Construir una estrategia de inclusión que recupere igualmente los cuidados como factor principal de la reproducción de la vida, que fortalezca el trabajo más comunitario, desarrollando actuaciones más abiertas y transversales que puedan atender a todas las personas en lo local, ofreciéndoles oportunidades de aprendizaje, relación y formación y, por supuesto, generando y promoviendo realidades de economía responsable social y solidaria.

\subsection{La vulnerabilidad en las puertas de las entidades públicas: los ayuntamientos}

La Ley vasca de Servicios Sociales de 2008 (art. 27) establece distintas atenciones a las realidades de la vulnerabilidad social (riesgo de exclusión) y de la exclusión social:

Los servicios integrados en el Sistema Vasco de Servicios Sociales se estructurarán entre los servicios sociales de atención primaria y los de atención secundaria. Los servicios de atención primaria atenderán, entre otras, las necesidades relacionadas con la inclusión social, con particular incidencia en la prevención de las situaciones de riesgo de exclusión social, garantizando la provisión y prestación de los mismos desde los servicios sociales municipales.

Por lo tanto, si queremos valorar las políticas públicas que atienden las situaciones de vulnerabilidad social, debemos apoyar y mirar a los dispositivos municipales encargados de acompañar este tipo de realidades. Es allí donde las personas viven y donde buscan en primera instancia protección e inclusión social. Razonablemente, los ayuntamientos necesitan reforzar los apoyos económicos y logísticos para canalizar estas realidades sociales crecientes con la actual crisis económica.

\subsection{La vulnerabilidad ante las puertas de las entidades del tercer sector guipuzcoano}

Las entidades del tercer sector en el ámbito de la intervención social tienen fundamentalmente presencia en el espacio de las personas que están en situación de exclusión social, y desarrollan su actividad acompañando estas situaciones de gran dificultad social. El modo en que las propias entidades definen su misión, así como los convenios con la administración pública que enmarcan los soportes para la exclusión, refuerzan esta labor de acompañamiento y apoyo a las personas en peor situación.

Sin embargo, las entidades también desarrollan programas y acciones centradas en la prevención de la exclusión y en la promoción de la inclusión social, programas que, sobre todo, se contextualizan en el ámbito de la activación del empleo con carácter formativo y ocupacional. Por lo tanto, podemos deducir que las entidades del tercer sector guipuzcoano más centradas en el ámbito de los programas de empleo atienden más situaciones de vulnerabilidad, aunque eso sí, dentro del estrecho marco de la inclusión por el empleo. En cambio, en las programaciones y memorias de las entidades sociales, en general, se incide en la atención a las personas más desfavorecidas en el ámbito de la exclusión, mientras que las acciones con las personas en situación de vulnerabilidad, tal y como encuadramos en este artículo, tienen poco peso.

\section{El desencadenante principal de la incertidumbre: el desempleo}

En nuestra sociedad, el empleo está construido como una de las dimensiones básicas de la persona: no sólo es la fuente principal de ingresos de la clase trabajadora, sino también un elemento de desarrollo personal, un mecanismo de participación e identidad social que organiza nuestros proyectos personales y da sentido a nuestra existencia. No obstante, esta definición construida en las sociedades occidentales es parcial, por dejar fuera del empleo todos aquellos trabajos fundamentales que realizamos, por ejemplo, en la esfera de la vida y del cuidado, o en la comunidad a través de la participación o de relaciones de solidaridad, que tejen redes que, a su vez, evitan la vulnerabilidad y la exclusión.

He aquí una primera brecha entre los trabajos y el empleo entendido como un marco cerrado y reduccionista, donde parece imposible dar cabida a toda la ciudadanía para proporcionarle los ingresos suficientes, y por lo tanto, los beneficios sociales que se derivan del empleo. A pesar de esta imposibilidad numérica del pleno empleo, y de que todos los datos a medio y largo plazo sobre el mercado laboral auguran una fuerte bajada de los puestos de trabajo en el sistema productivo, nuestra sociedad ha decidido mantener el empleo como pilar de acceso a la subsistencia, como mecanismo de pertenencia y participación en la sociedad, como contribución fundamental al Estado, y por lo tanto, como red principal de protección social ante las incidencias de la vida.

El empleo, definido como actividad que desarrollamos en el espacio público vinculado a un salario, también nos articula (bien o mal) la vida y sus tiempos, nos aporta una identidad social, nos permite ascender en la escala social, y nos da carta de ciudadanía, ya que, a través de él, contribuimos a la caja común de la Seguridad Social, que también repercute en derechos como la protección ante los acontecimientos de la vida, la enfermedad, la jubilación o la propia pérdida del empleo. Por lo tanto, en nuestras sociedades tener o no tener empleo fundamenta y determina, por lo menos hasta 
hace muy poco, la diferencia entre la estabilidad y el bienestar, y la exclusión y la pobreza.

Sin embargo, en el contexto actual de crisis, el empleo está perdiendo su peso social, por lo que no se puede considerar ya un elemento que evite la exclusión, o al menos, el riesgo de caer en ella. Precisamente algunos empleos han dejado de evitar la pobreza, y han dado lugar al fenómeno de personas trabajadoras que no dejan de ser pobres aunque trabajen y que, por lo tanto, no disponen de los beneficios que se le supone al mercado laboral y que ya hemos mencionado anteriormente. Como dice Imanol Zubero (2006: 11), se ha producido una transformación radical de la norma social del empleo.

Como esos icebergs que ocultan su volumen y muestran únicamente su punta, se van haciendo más presentes aquellos empleos que no ayudan a sostenerse, que dan una identidad precaria, que no ofrecen movilidad social, que no protegen de las incidencias, que restan ciudadanía y que desde luego no concilian con la vida ni con los cuidados de ésta. Una fuerte crisis económica ha puesto en jaque al pilar social que es el empleo, dejando algunos de estos empleos en un espacio social residual, y al Estado, sin una fuente importantísima de ingresos. Las transformaciones producidas en el mundo laboral durante los últimos años, como la deslocalización de las empresas, la división internacional de la producción o la revolución tecnológica, han ido reforzando la pérdida de valor de esa columna que ha sido primordial en la vida de las personas y en la construcción del Estado del bienestar.

El empleo como construcción cultural tiene un peso privilegiado en nuestras cartas magnas. Que figure en ellas le otorga el estatus de institución que regula y vertebra la relación entre la economía, y la vida y el desarrollo de las personas. Pero si este principio de relación entre empleo y subsistencia básica se rompe, ¿cuál es el plan de salida?, ¿cómo proveen nuestras cartas magnas su resolución?

\subsection{La zona más oscura del empleo: el desempleo estructural}

En Euskadi, podemos distinguir tres etapas diferenciadas respecto a las tasas de desempleo. La primera se desarrolló desde 1985 a 1996, y se caracterizó por una tasa de paro del $20 \%$ aproximadamente, como consecuencia de una profunda reconversión industrial. Una segunda etapa se extendió entre 2006 y 2008 , años en los que la tasa de paro llegó a bajar al 3,1\% (tercer y cuarto trimestre de 2007), pudiendo denominarse ese momento de pleno empleo. La tercera y última etapa sería la actual, en la que nos encontramos en la denominada crisis financiera mundial, con una tasa de paro del $11 \%$ en el segundo trimestre del 2017 (Eustat, 2017).

En la comparativa con otras comunidades autónomas del Estado, los datos del desempleo en nuestro territorio parecen positivos y nos crean cierta complacencia ('no estamos tan mal como otros') pero estamos dejando a más del $10 \%$ de la población de Euskadi descartada y sin acceso a través del empleo a una subsistencia mínima. Éste es uno de los tantos datos significativos que nos deben hacer reflexionar sobre las salidas que proponemos para las personas en cuanto a su protección, autonomía e igualdad de oportunidades.

\subsection{La zona en penumbra del empleo: las personas trabajadoras pobres}

Una de las herramientas principales para luchar contra la pobreza es el sistema de protección social, que palía la escasez de ingresos económicos y, por lo tanto, reduce la pobreza de personas y familias en nuestro entorno. Euskadi cuenta con un instrumento principal en esta lucha contra la pobreza, la renta para la garantía de ingresos (RGI), cuyo dato más significativo sobre el empleo y su capacidad inclusiva es aquel que nos indica que, en junio de 2017, el $20,1 \%$ de quienes cobraban esta prestación estaban trabajando y tenían ingresos por debajo o similares a la propia ayuda social (Lanbide, 2017).

Sin embargo, es importante resaltar el papel determinante que tiene el sistema de rentas mínimas ${ }^{1}$ en la prevención del avance de las expresiones más graves de la pobreza, a la vez que contribuye de forma decisiva a mitigar los efectos negativos de los procesos sociales y económicos asociados a la crisis de empleo posterior a 2008. Así, por ejemplo, este sistema llegaba en 2016 a un 69,3\% de la población en riesgo de pobreza ${ }^{2}$ (134.563 personas, un 6,3\% de la población total de Euskadi) y permitía reducir la distancia que separaba los ingresos disponibles respecto del umbral de pobreza de mantenimiento ${ }^{3}$ de un $74,2 \%$ a un $16,1 \%$ (Gobierno Vasco, 2016b). Esta política de garantía de ingresos ha permitido mantener a Euskadi muy lejos de los niveles de pobreza y desigualdad que caracterizan en la actualidad a todos los países de la Europa del Sur. A su vez, este sistema de protección sostiene una sociedad más estructurada, favorece la cohesión social y, por lo tanto, disminuye el conflicto social.

Aun y todo, las personas trabajadoras pobres son el primer colectivo vulnerable de esa ruptura entre la economía y el mercado laboral, un colectivo que tradicionalmente no se ha considerado excluido, pero

${ }^{1}$ Formado por la renta de garantía de ingresos (RGI), la prestación complementaria de vivienda (PCV) y las ayudas de emergencia social (AES).

2 "Las situaciones de riesgo de pobreza [...] se relacionan con la insuficiencia de ingresos de los hogares para hacer frente a las necesidades básicas (pobreza) o, en sentido más amplio, a las necesidades de gasto requeridas para el mantenimiento del nivel de bienestar mínimo esperado en una sociedad como la de la CAE [Comunidad Autónoma de Euskadi]" (Gobierno Vasco, 2016).

3 La pobreza de mantenimiento hace referencia a una situación de insuficiencia de recursos económicos para abordar de forma regular la cobertura de las necesidades básicas, particularmente las relacionadas con los gastos de alimentación, vivienda, vestido y calzado. 
que hoy día vive en una situación de inestabilidad, sin poder alcanzar los ingresos mínimos necesarios para la subsistencia y, en consecuencia, necesitan acudir al sistema de protección público. Así lo recoge la reciente Encuesta de Pobreza y Desigualdades Sociales 2016 (EPDS), cuando afirma que el desempleo pierde "algo de importancia" como principal determinante de la pobreza, aumentando la situación de pobreza en los hogares de población ocupada.

Contradictoriamente, en 2016, un contexto donde hay más empleo debido a la recuperación macroeconómica, repunta respecto a 2014 la población ocupada que está en situación de pobreza real. El empleo precario es el principal factor vinculado al incremento de las tasas de pobreza que se observa entre 2014 y 2016 en los hogares de personas ocupadas. Este hecho también se apunta en la introducción de la memoria de 2016 del Consejo Económico y Social Vasco: la baja intensidad y la inestabilidad laboral siguen avanzando y comienzan a engrosar el fenómeno de la pobreza.

Este segundo dato nos indica que se está rompiendo el vínculo entre la recuperación económica y el empleo también en Euskadi y que el desarrollo económico (el dinero) y el mercado laboral (las personas) van distanciándose, creándose una relación de mayor desigualdad.

\subsection{Las otras sombras del empleo actual}

Si profundizamos en los datos de ocupación de la población activa del último año, observamos que el empleo que se crea en la actualidad, por su temporalidad, modo de contratación, salario y otras características, es un empleo precario, que dista mucho de aquel concepto que nos articulaba la vida, nos sostenía y nos promocionaba dentro de la escala social.

Desde los años setenta, las causas que pueden explicar este nuevo escenario económico, social y, por supuesto, político, son fenómenos como la globalización, que ofrece a la economía posindustrial una mesa de juego más amplia, permitiendo la deslocalización de la producción y la división internacional del trabajo, impulsando la competencia entre países y forzando a la baja los salarios. Una economía en la que cada día pesa menos la producción, para tener un peso mayor la economía financiera especulativa, que, junto con la revolución tecnológica, ha transformado el mundo de las fábricas y el empleo, aumentando considerablemente los niveles de paro estructural y de precarización, con contratos basura y mano de obra barata en nuestras sociedades europeas.

Esta economía ha creado un mercado laboral dual, donde cae la ocupación de baja y media cualificación profesional, mientras que asciende la de mayor cualificación. Es decir, algunos sectores financieros y tecnológicos tienen oportunidades impensables en otras épocas y, por el contrario, un buen número de personas trabajadoras pierden, empujadas hacia la exclusión con trabajos de poca calidad, salarios muy bajos (Pérez Moya, 2016: 480). Se ensancha así la brecha salarial y de condiciones laborales entre estos colectivos.

La población trabajadora activa también se enfrenta a las sombras de otras tendencias, como la inestabilidad laboral, la temporalidad, las dificultades de ascenso profesional o el declive de las reivindicaciones colectivas. La inestabilidad es uno de los rasgos característicos del empleo actual. La intermitencia en la sucesión de contratos diseña trayectorias laborales fragmentadas, sobre todo en grupos sociales como las personas jóvenes o las mayores de 45 años. Para la historia quedan los contratos indefinidos, que no se dan ni en el inicio de la relación contractual, ni tras una andadura más o menos larga de ésta. Así, encadenar multitud de contratos temporales se ha convertido en una práctica habitual, que reduce el objetivo de la estabilidad laboral a la no inactividad. Esta situación de inestabilidad laboral provoca incertidumbre vital y somete las personas a decisiones vitales tomadas con una mirada cortoplacista a la hora de marcar sus objetivos personales.

En la Comunidad Autónoma Vasca, el porcentaje de población asalariada con contrato temporal ha pasado del $21 \%$ en 2009 al $26,4 \%$ en 2016 , marcando una tónica ascendente (Gobierno Vasco, 2016a: Tabla 2.3a). De la población activa y con contrato, el $25,9 \%$ de los hombres tienen contrato temporal, frente al $27 \%$ de las mujeres; sin embargo, las mujeres de entre 30 y 44 años en esa situación sobrepasan en 4,7 puntos a los hombres situados en la misma franja de edad (30,2 \% frente a $25,5 \%$ ).

La dificultad de alcanzar una estabilidad laboral entre las personas jóvenes, e incluso entre las adultas, conlleva, en la gran mayoría de los casos, retrasar decisiones en el ámbito de la vida. Hablamos de personas que no pueden emanciparse o parejas que no pueden hacer un proyecto en común, incluso parejas o mujeres que deciden posponer la paternidad/maternidad ( $30,2 \%$ de las mujeres de 30 a 44 años con contrato temporal) o reducir el número de hijos e hijas para no sufrir una situación de pobreza leve o moderada.

La Encuesta de Necesidades Sociales de 2014 también detecta, respecto a encuestas anteriores, un repunte de los problemas vinculados a la posibilidad de tener el número de hijas e hijos deseados, debido a la situación económica y laboral y a las dificultades para el cuidado de la descendencia. Así, en 2014, el $5,6 \%$ de los hogares de la CAV declaraba tener problemas para alcanzar el número de hijos e hijas deseado. Y ese mismo año, la población que deseaba independizarse del hogar de origen y no podía hacerlo por motivos económicos alcanzaba el $50 \%$ en la franja de edad comprendida entre los 25 y 34 años (Gobierno Vasco, 2016a: Tabla Rep-2). Por otro lado, 
una gran parte de las personas contratadas lo había sido varias veces durante el año y la duración media de los contratos temporales era de 48 días (Consejo Económico y Social Vasco, 2017).

La temporalidad es otra de las sombras crecientes que resta al empleo sus considerados beneficios. En 2016, el porcentaje de personas ocupadas a tiempo parcial en Euskadi era del $17,7 \%$, ratio superior a la del conjunto del Estado. El 40,1\% de los contratos eran a tiempo parcial. Por sexo, el peso de la contratación a tiempo parcial es muy distinto, pues representa más de la mitad de los contratos firmados por mujeres (53,1\% en 2016), frente al $26,8 \%$ de los firmados por hombres (ibídem).

A partir de los datos de la inestabilidad, temporalidad y parcialidad, podemos definir a las mujeres como otro colectivo vulnerable, ya que tienen un mayor número de contratos parciales y no indefinidos. A esta peor situación laboral, puede añadirse, sin duda alguna, la precarización del cuidado de la vida, ante la falta escandalosa de apoyos en el ámbito doméstico y de otro modelo económico más sostenible y respetuoso con aquélla. En este sentido, es significativo el dato de 2015 que muestra que las mujeres que trabajan fuera de casa dedican un $50 \%$ más de horas diarias al cuidado de hijos/as menores y de personas dependientes que los hombres, y hasta un $75 \%$ más de tiempo a las tareas del hogar (Eustat, 2016).

Los salarios son otro indicador claro de la precarización del empleo. En 2016, el 24,9\% de la población asalariada de Euskadi tenía un salario variable. Este porcentaje se ha multiplicado por dos (11,2\%) desde 2013 y está marcando una clara tendencia en ascenso. Los empleos con salarios fijos, en cambio, han tendido a la baja, pasando de un $88,8 \%$ en 2013 a un 75,1\% en 2016 (Gobierno Vasco, 2016a: Tabla 2.9b). Además, casi uno de cada cuatro salarios tiene un complemento variable, dato que no hace más que incidir en la inestabilidad y precariedad de las condiciones laborales, haciendo muy difícil la organización económica doméstica y la conciliación de la vida, al quedar ambas al albur de las dinámicas de la actividad laboral.

La promoción social a través del empleo también resulta cuestionada por dos de los aspectos que aparecen en la Encuesta de Condiciones de Trabajo de 2016. Por una parte, la tasa de promoción en el trabajo ha descendido del 30,5\% en 2009 al $24,7 \%$ en 2016. Si segregamos estos datos por sexo, vemos que los hombres tienen una probabilidad de promoción ocho puntos superior a la de las mujeres (28,7\% frente a $20,6 \%$ en 2016 , respectivamente) [Gobierno Vasco, 2016a: Tabla 2.8b]. Así, el 65,1\% de la población asalariada y asimilada se ha mantenido en la misma posición en la empresa durante 2016 (10 puntos por debajo que en 2013) [ibídem: Tabla 2.7b].

Por otra parte, la principal vía de acceso al primer empleo, según la Encuesta de Inserción Laboral de Lanbide de 2015 realizada a personas estudiantes recién licenciadas o graduadas de la Universidad del País Vasco (UPV/EHU) en 2012, fueron la familia, las amistades y los contactos ( $24 \%$ de los casos). El círculo social, que mueve un volumen importante de empleos, es reducido y totalmente discrecional, ya que normalmente las redes sociales son fruto de compartir espacios vitales similares (barrio, colegios, ámbito laboral) y suelen estar compuestas por personas con un nivel económico, cultural y social similar. Aparece, pues, con más fuerza lo que se ha denominado posición social heredada y, por consiguiente, se reducen los otros mecanismos de movilidad social, promoción o mejora en la calidad de vida, y también la capacidad que tiene el empleo para alcanzar ese fin.

Otra de las sombras de las condiciones actuales del empleo y de sus rupturas se encuentra en la dificultad de mantener una conciencia colectiva reivindicativa: según el Consejo Económico y Social Vasco, a comienzos de 2017 tres de cada cuatro trabajadores tenían sus convenios colectivos pendientes de renovar o recuperar, y prácticamente sólo uno de cada diez trabajadores gozaba de un convenio actualizado.

\subsection{Otras sombras sociales de nuestro entorno: la desigualdad crece}

Echando una mirada a los datos de pobreza y desigualdad en Euskadi (Gobierno Vasco, 2016b), el deterioro asociado a la crisis muestra en 2016 una Euskadi más desigual que en 2012, con incidencia particular en los grupos más pobres. Se muestra una polarización y alejamiento entre los grupos con mayores ingresos y los que se sitúan en el 10\% más desfavorecido de la población. Así, alrededor de un $51,5 \%$ de la población que es atendida dentro del sistema de protección social pero que no consigue salir de la pobreza real (el 16,1\% en 2016) y de las personas que se encuentran en una situación de pobreza real y que no acceden al sistema (el 22,5\%) señalan no disponer de ingresos suficientes para hacer frente a las necesidades básicas del hogar. Se observa una estabilización y, en ocasiones, un incremento de los indicadores relacionados con la cobertura de las necesidades básicas en estos colectivos (ibídem). Por último, si bien cae la pobreza infantil, aumenta, entre los colectivos pobres, la presencia de población nacional y de hogares con población ocupada.

\subsection{La pobreza encubierta: la precariedad vital}

En 2016, la Encuesta de Pobreza y Desigualdades Sociales se lanza a examinar algunas situaciones inquietantes que están en el trasfondo de todo lo que hemos mencionado: la pobreza encubierta entre la población de 18 a 44 años. De esta manera, analiza la dificultad experimentada por algunas personas, principalmente jóvenes, para acceder a una vida independiente por falta de recursos económicos suficientes. 
La encuesta detecta 100.164 personas de entre 18 y 44 años que no cuentan con los recursos suficientes para emanciparse del hogar de origen. En 2012, eran 95.754, de modo que ha habido un ascenso. Sobre el total de la población de esa edad, quienes carecen de recursos para emanciparse repuntan en 2016 hasta el $14,4 \%$, por encima del $12,4 \%$ de 2012 y también del $12,9 \%$ de 2008 .

El perfil sociodemográfico de este colectivo es el de una persona de entre 25 y 44 años, con estudios cualificados, que está en desempleo o tiene una ocupación inestable. Además, la precariedad laboral figura en la encuesta como el principal motivo señalado para no emanciparse, por delante de la ausencia de vivienda.

\section{Ante un nuevo plan vasco de inclusión activa: propuestas}

Iniciamos este artículo aludiendo a Ulrick Beck y a su metamorfosis del mundo (2017), obra que resume a la perfección la idea, el sentimiento que queremos trasladar en este artículo. Vivimos en un mundo cada vez más difícil de entender. No sólo está cambiando: está en plena metamorfosis. La metamorfosis implica una transformación mucho más radical, en la que las viejas presunciones de la sociedad moderna se derrumban y surge algo distinto, nuevo. Para entender esta metamorfosis del mundo, hay que explorar nuevos principios, fijarse en qué permanece de lo viejo, y buscar estructuras y normas futuras en la confusión del presente. En resumen, los riesgos sociales explicados en los párrafos anteriores han llegado para quedarse; las transformaciones del empleo y del mundo de la economía están marcando y desplazando el binomio subsistencia-empleo, así como los beneficios atribuidos a éste en las últimas décadas.

En nuestro territorio, los datos y su evolución apuntan - a su ritmo, pero de forma imparable- a que otras relaciones con la subsistencia, la economía y el empleo son imprescindibles para la vida social y pública a medio y largo plazo. Las personas necesitamos otras respuestas, otros contextos más posibilitadores que no nos pongan a competir por los recursos para la subsistencia, ya que la competencia aumenta la conflictividad, la percepción de amenaza y la sensación de inseguridad, y refuerza argumentos de 'primero, lo nuestro', quebrando un principio fundamental de nuestra democracia: la solidaridad.

En esta búsqueda de salidas y de nuevos marcos conceptuales para otro imaginario social que aporte -como el empleo-subsistencia, identidad, participación, protección y movilidad social, los futuros planes de inclusión social necesitan cierta dosis de profecía y de valentía política y técnica, y una estrategia de gobernanza participativa:

- El primer elemento de la inclusión: la protección. Cualquier plan de inclusión, o cualquier actuación pública para mejorar la situación de la ciudadanía, tiene que cumplir con este elemento vertebrador de todas las demás acciones. Esa protección podrá venir del empleo, pero en aquellas situaciones en que no lo haga, o en que lo haga mal, el sistema tendrá que amparar la vida y el proceso que pueda surgir a partir de ella.

- El sujeto vulnerable que presiona. De diferentes maneras y con diferentes discursos, las personas en situación de vulnerabilidad también reclaman lugar y reconocimiento en los sistemas de protección y en las estrategias de inclusión. No parece, por los datos disponibles, que podamos eludir en los próximos años políticas públicas que vayan a atender y sostener también a las personas que se sienten vulnerables. De no atender esta demanda, esas situaciones presionarán en otras direcciones, quizás cuestionando las políticas públicas y rompiendo con el principio de solidaridad.

- Por lo tanto, planes de igualdad social. La desigual relación social con la economía, la esfera de la vida y el mercado laboral que está marcando los espacios de vulnerabilidad y precariedad creciente que viven las personas de nuestro entorno requiere una estrategia social y política de igualdad social y, por lo tanto, inclusiva. De esta manera, los planes de inclusión se reconvierten en planes universales que posibilitan la mejora de la ciudadanía allí donde se necesitan (como otros sistemas públicos) y abren el sujeto de su acción a la comunidad, al mundo y también al espacio empresarial, generando allí espacios de amparo institucional.

- Más de un sujeto en la acción inclusiva: la comunidad y la empresa. Dicho plan de inclusión social, además, debe ampliar los sujetos de su acción, sujetos vinculantes a la dinámica inclusión-exclusión, como son la comunidad y la empresa, ya que es en la dinámica relacional persona-comunidad-empresa donde se produce la inclusión o la exclusión. Los sujetos de los planes y de sus acciones serán las personas y sus capacidades, y también la comunidad y sus capacidades, y las empresas y sus capacidades.

- La inclusión, más que el empleo. Además, las estrategias inclusivas necesitan desmarcarse del empleo como única vía de salida, sumando otras acciones que articulen e impliquen otros mecanismos del contexto social de mayor incidencia que la empleabilidad del sujeto, como poner en valor los cuidados de la vida, el aprendizaje o los espacios colaborativos. Impulsemos el trabajo asociativo y ciudadano que colabora, revitaliza y reivindica los espacios de reflexión, diálogo, contraste y acción, también en los planes de inclusión. El empleo es una construcción cultural fuertemente arraigada, y será difícil destronarla de su altar y del papel principal que le hemos otorgado, pero nuestro modelo económico se agota en él, se distancia de los puestos de trabajo, de la mano de obra. Tarde o temprano muchas personas no contarán con 
un lugar en la esfera laboral, y ello constituye un problema al que tendremos que responder.

\section{Las estrategias de Caritas ante la vulnerabilidad}

Como muchas otras entidades, también Caritas hace una lectura de las realidades sociales que nos rodean $y$, a partir de ellas, diseña estrategias y actuaciones que mejora las condiciones de vida de las personas y los pueblos del mundo. Aun no siendo el objeto del artículo, por coherencia con él, nos parece importante aportar tres estrategias institucionales que van en la línea de las propuestas que hemos hecho al Plan Vasco de Inclusión 2017-2021:

- Los derechos sociales. Caritas diseña campañas de sensibilización y concienciación que pretenden, con sus lemas, "promover el cambio social, tanto en las estructuras como en los mecanismos y cimientos que lo sustentan, para lograr la realización de la revolución del amor" (Benedicto XVI, 2015: 31). La Iglesia nos llama a un compromiso social, "un compromiso que sea transformador de las personas y las causas de la pobreza, que denuncie la injusticia, que alivie el dolor y el sufrimiento y que sea también capaz de ofrecer propuestas concretas" (Cáritas Española, 2017). Esta concienciación es una labor personal y colectiva que busca el reconocimiento de que todas las personas que viven en este planeta tienen derecho a una vida digna (Cáritas Española, 2010).

- Refuerzo de la comunidad. En la misión de Caritas, la comunidad, insertada en el barrio, en la ciudad, es la base principal para la articulación de actuaciones con las personas. Somos y vivimos en comunidad, y a ella estamos convocadas las personas. Es una invitación a tejer red con los demás; es una llamada a aprender a vivir en común, a respetar y hacer posibles los derechos humanos de todas las personas, y a convivir de una manera sostenible en la casa común que nos acoge a todas las personas.

- Economía solidaria. Cambiando el foco, cambiamos los resultados. "También en la vida económico-social deben respetarse y promoverse la dignidad de la persona humana, su entera vocación y el bien de toda la sociedad. Porque el hombre es el autor, el centro y el fin de toda la vida económico-social" (Pablo VI, 1965: 63). En este sentido, Caritas está impulsando el desarrollo de empleo inclusivo, reforzando empresas de inserción, promoviendo el comercio justo y el consumo responsable, y diseñando una estrategia de finanzas éticas.

\section{Dos historias de vida relacionadas con las situaciones de vulnerabilidad}

A continuación, expondremos dos historias de vida que nos acercarán a la realidad de los procesos sociales de tránsito entre las distintas zonas de la inclusión/exclusión social. Además de poner rostro a estas situaciones, nos servirán para buscar deficiencias y oportunidades en las políticas sociales de prevención y atención a colectivos en riesgo y exclusión social. Las palabras de estas personas podrán ayudarnos a mejorar estas políticas sociales, tanto públicas como de la iniciativa social ${ }^{4}$.

Ofrecemos dos historias de vida ${ }^{5}$ de dos personas con trayectorias bien distintas. Una, ocurrida en la década de 1980, en la que una mujer y su familia entraron en la zona de vulnerabilidad y consiguieron salir, para 'regresar' a la zona de inclusión. Otra, de un varón que a comienzos del siglo XXI se acercó a la zona de vulnerabilidad hasta caer en la zona de exclusión. Relacionaremos las historias de vida con los contextos sociales en los que se produjeron ${ }^{6}$.

\subsection{Historia de vida de A.}

Varón nacido en la primera mitad de la década de 1960 en una de las capitales vascas. La mayor parte de su vida la ha vivido en la capital en la que nació. Cuenta con un título de formación profesional de grado II, y entre diez y veinte años trabajó con contratos laborales y de interino en la administración pública. En 2000-2001, tuvo su primer contacto con el mundo del sinhogarismo, al dormir dos meses en la calle. En 2011, tras un largo proceso de vulnerabilidad social, pasó a situación de sinhogarismo.

Hasta la muerte de su madre, vivió en casa de su padre y su madre, mientras trabajaba en distintos destinos de la administración pública. Estuvo en la zona de integración social - con algunas características de vulnerabilidad, por carecer de trabajo fijo-. Tuvo empleos, mantenía relaciones familiares y de amistad, y participaba en distintas actividades deportivas y del asociacionismo vecinal. A principios de la primera década del siglo XXI, como consecuencia de la muerte de su madre, su situación comenzó a ser más frágil y precaria, y pasó a la zona de vulnerabilidad.

${ }^{4}$ Las dos personas accedieron a relatar su historia vital como parte de este artículo en torno a los procesos de inclusión, vulnerabilidad y exclusión social. Las entrevistas en profundidad se realizaron en junio de 2017.

${ }^{5}$ El planteamiento de las historias de vida ha seguido las aportaciones de Bertaux (1997). Este autor entiende la investigación de relatos de vida a través de la técnica de la entrevista narrativa, en la que el investigador solicita al sujeto entrevistado que cuente toda o parte de su experiencia vivida, en nuestro caso, el itinerario vital a través de las zonas de inclusión, vulnerabilidad y exclusión social. El objetivo de esta narración es construir una serie de hipótesis plausibles que permitan encontrar en las biografías las 'huellas' de los contextos y procesos sociales que posibilitan comprender las trayectorias vitales.

${ }^{6}$ En la línea de Mills (1959), hemos pretendido captar la historia, la biografía y la relación entre ambas, buscando las intersecciones entre la biografía, la historia y la sociedad. 
Al morir su madre, comenzó con síntomas depresivos y se acentuó la mala relación que tenía con su padre, al que describe como autoritario. Con el tiempo, se enfadó con su única hermana y perdió la relación. Su madre mediaba en los conflictos con su padre, y al fallecer aquélla, la convivencia y la relación con el padre empeoró hasta hacerse insoportable: "cuando murió mi madre, mi mundo organizado se vino abajo".

En varias ocasiones, esa mala relación le llevó a tener que abandonar el domicilio familiar. Además, al comenzar su padre una nueva relación de pareja, la comunicación con él llegó a ser casi nula. En su discurso, relata que nunca llegó a superar la muerte de su madre y que incluso le llevó a una depresión.

La primera vez que cobró la renta de garantía de ingresos (RGI) fue en 2001-2002, cuando vivía en casa de su padre (su madre ya había fallecido) y estaba esperando un destino laboral en la administración pública. De esta ayuda social le hablaron en el Inem cuando se le acabó la prestación por desempleo. En esas fechas, tuvo que irse a trabajar fuera, porque no encontraba destino en su provincia ni en su comunidad autónoma de origen. Después de unos meses esperando sin éxito un puesto en la administración pública de su provincia o de la CAV, le ofrecieron una plaza en una gran capital española a cerca de $500 \mathrm{~km}$ de distancia de su entorno conocido.

En el nuevo destino, vivió unos cuatro años. Allí se acentuó su depresión y entró en zona de aislamiento social. No tenía ni amigos ni familia. De vez en cuando, salía por la noche a beber y alternar con personas que conocía por el camino. Vivió en esta gran ciudad hasta que le quitaron un plus de productividad que estaba cobrando y su situación económica se hizo mucho más precaria. Pasó de cobrar unos $1.300 €$ a cobrar $700 €$, con el pago de una vivienda en alquiler de $450 €$. Su salud mental seguía resquebrajándose (depresión), aislado en una gran ciudad y con un sueldo menguante que difícilmente le servía para mantenerse. Los anclajes para hacer frente a su vulnerabilidad desaparecían.

En vistas de la situación, su hermana le propuso que dejara el empleo y se instalara en su casa con su familia, en la misma ciudad donde ambos habían nacido y criado (año 2006-2007). Allí comenzó a sentirse un "estorbo"; su hermana le daba algo de dinero para sus pequeños gastos. Acudió al centro de salud mental y comenzó a tomar antidepresivos. Fue perdiendo relación con sus amigos, a los que su hermana había llamado para que intentaran 'sacarle a la calle' ("ser amigo para cargar con alguien..., pues no").

A través del centro de salud mental, no entró en contacto con los servicios sociales, ni le derivaron a ellos. No le ofrecieron tratamiento psicológico, pero "tampoco creía en los psicólogos”. En el centro, le atendían en una sesión cada dos meses. En esa época, no solicitó ayudas económicas, aunque sabía de la existencia de la renta de garantía de ingresos y de los servicios sociales?.

Por desavenencias con su hermana, dejó su casa y vivió un par de meses en la calle. Fueron los policías municipales quienes le hablaron del Albergue Municipal $^{8}$. En esos meses, señala que ni siquiera supo dónde estaba Aterpe (centro de Caritas de atención a personas sin hogar): "qué más hubiese querido yo saber que por allí hubiera podido comer..., que por lo menos hubiera podido comer un bocata". Relata que comía de lo que encontraba en los contenedores de basura.

En esa etapa, A. tomó contacto con el mundo de la calle, durmiendo en parques, comiendo de los contenedores de basura y en ocasiones socializando con algunas personas migrantes, también en situación de sin hogar. Tras un par de meses en la zona de exclusión, la abandonó para regresar a la zona de vulnerabilidad cuando pudo volver al domicilio de su padre (quien se fue a vivir con su nueva pareja a otra capital de provincia española), manteniéndose con un empleo precario y, posteriormente, con el cobro de la herencia familiar tras el fallecimiento de su padre y la posterior venta de su piso.

Entre 2009 y 2010, en plena crisis económica, vivió solo en el domicilio propiedad de su padre. Encontró trabajo en un bar, en unas condiciones laborales muy malas: comenzó trabajando los fines de semana por un salario de 25 € diarios y llegó a trabajar a jornada completa por unos $500 €$ mensuales.

En 2011-2012, su hermana y él vendieron el piso familiar de la herencia y se repartieron el dinero. Sin embargo, no estuvo de acuerdo en el reparto, se enfadó con su hermana y rompió la relación. Con el dinero, y tras abandonar el piso vendido, se fue a vivir de alquiler a un piso del mismo municipio. En esa época ya casi no tenía relación con amigos: ni le ofrecieron ayuda ni él la pidió. Ahí estuvo unos cinco años, hasta que se le acabó el dinero. Pagaba $840 €$ al mes de alquiler. Reconoce una mala administración del dinero, sobre todo al principio. Desde que cobró el dinero de la herencia hasta que se le agotó, encontró algunos trabajos eventuales. Los buscaba, pero no los encontraba ("ya tenía, 49-50 años y se me estaba haciendo muy difícil encontrar trabajo").

${ }^{7}$ Queremos destacar a los centros de salud como lugares en los que profesionales atienden a personas en situación de vulnerabilidad social. Desde su enfoque biopsicosocial, están capacitados para conocer situaciones de fragilidad emocional y social, y derivar el caso a las trabajadoras sociales del servicio o a de los servicios sociales de base. En esos centros de salud, se pueden comenzar intervenciones preventivas con casos de vulnerabilidad de personas que no han llegado al umbral de la exclusión. Poniendo el acento y los recursos en estos espacios, se implanta una política social preventiva al estilo de la que se realiza en los colegios con los menores en riesgo de desprotección.

${ }^{8}$ De esa época, relata malas experiencias con los policías municipales, a los que atribuye escasa formación y manejarse únicamente con el autoritarismo. 
En los meses anteriores a cobrar la herencia y en los años posteriores, trabajó en seguridad, en un bar y seis meses con un contrato municipal de fomento de empleo, como jardinero. Empleos precarios que le hacían continuar en la zona de vulnerabilidad, a pesar de haber cobrado la herencia. No pensó en pedir ayuda al sistema, a los servicios sociales ("no, porque me podía valer por mí mismo; pido ayuda si no tengo, no cuando tengo"). Estaba ya preparado para pasar a la zona de exclusión. El 'clic' fue agotársele el dinero de la herencia.

Vivió de alquiler hasta que, en 2014, se le acabó el dinero de la herencia. Tuvo que abandonar el piso por desahucio. No tenía medios económicos para enfrentarse al pago del alquiler y no respondía a las cartas que le reclamaban el pago de lo adeudado. No pidió ayuda a ningún servicio: "no sabía ni por dónde empezar [...] servicios sociales, lo veía por televisión..., yo pensaba que ayudaba a extranjeros, básicamente". No se puso en contacto con Stop Desahucios; los conocía, pero pensaba que no le podían ayudar ("mi caso era diferente..., yo me quedé de okupa al no poder pagar el alquiler”). Ni siquiera se enteró de si había algún profesional que le pudiera ayudar en todo el proceso. Se quedó fuera, sin información, pensando que nada le correspondía, con un estado de ánimo bajísimo, sin apoyo social y sin saber si le correspondía algún derecho para hacer frente a su situación.

Señala que, con el dinero que le dejó un amigo, se fue a una pensión, "dándole vueltas a la cabeza: a partir de ese momento, ¿qué?”. Y cuando se le acabó el dinero, recurrió al Albergue ("allí me fui directo"). Lo conocía de su anterior estancia, de cuando vivió un par de meses en la calle. Estaba de nuevo en la zona de exclusión o marginación, pero había una diferencia entre la primera y la segunda vez que estuvo en el Albergue. La primera vez, nos explica, “había una puerta... (volver a casa de mi padre o casa de mi hermana); esta vez, no había puerta... Insistí bastante, o sea, [yo soy] una persona de Donosti, que siempre ha trabajado aquí, excepto temporadas..., lo único que se me ofrecen son tres puñeteros días y luego la calle [...]. Me dijo, ¿por qué no vas a hablar con Caritas?".

En esta situación, también se dirigió a los servicios sociales para preguntar por la renta de garantía de ingresos. La solicitó, pero se la denegaron, porque consideraron que no cumplía los requisitos, al haber recibido una herencia hacía unos años ${ }^{9}$. En los servicios sociales del Ayuntamiento, le podían haber dado ayudas especiales para pagar el alquiler de una vivienda o habitación, pero para eso necesitaba contrato y empadronamiento. Lo buscó, pero conseguirlo era muy complicado. Dice que estuvo un año sin encontrar nada ("un año, bastante más

9 El sistema no entraba a valorar su situación de necesidad, sus dificultades anteriores, si había hecho un uso adecuado del dinero recibido...; únicamente, que no entraba en el baremo del dinero ingresado y del tiempo transcurrido. de un año [...], llamando a un montón de números de teléfono, que eran a los que llamábamos todo el mundo [...]. Se mueve muy poquito piso, y el que se mueve es para sacarle dinero"). Relata que le dijeron que interesaba para alquilarlo un fin de semana "a un guiri”.

Estuvo en Hotzaldi (alojamiento nocturno de Caritas). Luego, en estancia especial en el Albergue Municipal, a la espera del cobro de una ayuda social. Tuvo que dejar el Albergue cuando le denegaron la renta de garantía de ingresos; le reclamaban papeles, "que, obviamente no podía presentar [...]. Un fin de semana, con alevosía, me dejaron en la calle [...]. ¡Cómo quieres que te enseñe papeles, si lo único que llevo en la mochila son cuatro cosas!, lo único que he podido sacar de la casa... [...] Me pusieron en la calle... [...]. El único recurso que me quedó fue el SMUS [Servicio Municipal de Urgencias Sociales] y [la] plaza en el Gaueko [alojamiento nocturno municipal]". Conoció la mayoría de los dispositivos para personas sin hogar: Albergue Municipal, Hotzaldi, comedor social, centro de día y sala de noche de Aterpe, además del Gaueko.

En la actualidad, A. está en un centro residencial para personas en situación de exclusión cronificada, realizando un proceso de inclusión social. El centro pertenece al tercer sector, ocupa una plaza conveniada con la Diputación Foral (financiada completamente por esta institución), que fue la que le derivó, vía servicios sociales municipales.

\subsection{Historia de vida de $M$.}

Mujer nacida en la primera parte de la década de los años cuarenta en un pueblo andaluz. Junto con su padre, madre y hermanos y hermanas, emigró al País Vasco con 12 años. Sin estudios, trabajó desde muy temprana edad en los negocios familiares. Con 14 años, empezó a trabajar de operaria industrial en una gran empresa vasca, hasta que, al casarse, dejó la empresa para trabajar como 'ama de casa'. Tuvo tres hijos e hijas. Separada de su marido después de 26 años de matrimonio, cuando hijos e hijas habían alcanzado la mayoría de edad. En los primeros años de los ochenta, ella y su familia atravesaron una situación de vulnerabilidad social que superó con el contrato laboral fijo que consiguió como limpiadora en el ayuntamiento de su localidad.

El marido de M. tenía una pequeña empresa de mecanizados que creó hacia 1975-1976, cuando en España comenzaban a sentirse los primeros golpes de la crisis económica de 1973. El taller nunca llegó a despegar ("en mi casa, se vivía como un obrero medio-bajo"). En el hogar, las estrecheces económicas fueron en aumento: "había que hacer presupuestos bajos para que le dieran el trabajo [...]. Cada vez entraba menos dinero en casa, llegábamos justo, justo [...]. En esa época se cerraron muchos talleres pequeños, sí”. 
En plena crisis económica, con destrucción de empleo y aumento de las tasas de desempleo, la pequeña empresa se pudo mantener hasta 1981. Mientras que los años anteriores la situación familiar presentaba síntomas de vulnerabilidad, con esas estrecheces económicas y el riesgo de cierre del negocio, al cerrarse éste, la situación se tornó dramática: ni el padre ni la madre tenían empleo remunerado, ni a ninguno de los dos le correspondía prestación por desempleo. Después de años de estrecheces, en el hogar no había dinero ahorrado.

Con deudas con proveedores y con la Seguridad Social, la Inspección de Trabajo decidió cerrar el taller. En la familia, no había dinero ahorrado, los bienes de la empresa se embargaron y nada correspondió al empresario, que únicamente consiguió quedarse con unas 180.000 pesetas. Sin empleo, sin dinero, sin ahorros, sin prestaciones económicas, en plena época de destrucción de empleo, la familia se encontraba en la zona de vulnerabilidad social.

Podían contar con el apoyo de las familias de origen, con las que mantenían relación. A pesar de ello, las palabras de la entrevistada muestran la desesperación: ella tenía hermanos que no le iban "a dejar pasar hambre". De esas 180.000 pesetas, ella se quedó con 30.000 para los gastos de manutención propios y de sus hijos e hijas; el resto se lo quedó el marido para intentar montar un negocio en otra provincia española. Ante las estrecheces económicas ("esas 30.000 pesetas, estirando todo lo que se podía"), comenzó a buscar trabajo: "cuando las vecinas me veían levantándome temprano, comprando el periódico y buscando trabajo..., me encontraron a una señora para cuidarla".

Esas vecinas que la ayudaron a buscar ese primer empleo eran mujeres muy conocidas de la entrevistada, con las que había compartido tiendas de comestibles del barrio y parques mientras cuidaban de sus hijos e hijas. La densidad social, un barrio con una parroquia activa y con una asociación de vecinos con mucho empuje. Barrio de los años setenta, activismo político y solidaridad vecinal, barrio de unos 3.000 habitantes de clase obrera que creció en la época del desarrollo industrial vasco de los años sesenta.

Esas mujeres conocidas frecuentaban la parroquia. Enseguida se pusieron a intentar ayudarla, sabían de su situación ("para entonces [entre] ellas ya habían hablado"). Consiguió ese primer empleo precario: "con Carmen, iba por las mañanas..., luego iba..., le daba la cena...". Limpiaba la casa y cuidaba a esta persona mayor. Fue el primer trabajo desde el cierre del taller; estuvo un tiempo, pero tuvo que dejarlo, porque le pedían que pasara las noches y "no quería ni podía dejar a mis tres hijos pequeños solos".

De esos momentos, ella nos relata: "me acuerdo [de] que una vez vino Gotzon, el cura del barrio. Yo estaba planchando. 'M., ¿qué tal estás?', y cuando se fue, me metió dinero en la tabla de planchar. Me sentí muy mal. 'Gotzon, esto no, esto no, yo lo que quiero es trabajar. Yo tengo hermanos, y yo sé que a mí no me van a dejar pasar hambre, pero...". Se conocían de años de convivencia en el barrio. Por esos días, otra mujer que no conocía se puso en contacto con ella, "muy buena mujer..., de Caritas".

Nunca ha llegado a saber exactamente cómo esta mujer llegó a conocer su situación, pero probablemente fue por las vecinas. Tampoco se presentó como miembro de Caritas, aunque con el tiempo entendió que era una voluntaria de esta entidad: "una bellísima persona, tanto ella como su marido. [...] Yo creo que me hizo una llamada, que sabía cómo estaba...”. Esta mujer la acompañó a los servicios sociales municipales, de los que nunca había oído hablar. De allí surgió la oportunidad de encontrar un contrato indefinido de trabajo, que mantuvo hasta su jubilación. Ni sabía lo que era Bienestar Social ni sabía dónde se encontraba, porque como dice ella, "yo antes estaba más metida en mi casa [...] luego ya, cuando salió ese trabajo..., ¡bendito de Dios!”.

Con "muy buenos informes de la familia de la mujer [a la que había cuidado]", se dirigieron a Bienestar Social. "Y, entonces, esta señora, Milagros, me llevó a Bienestar Social y habló con la jefa, y dijo, 'esta mujer necesita trabajo, con sus tres hijos, tenemos muy buenas referencias de ella [...], lo que necesita es un trabajo fijo, por la Seguridad Social y todo...".

Relata que, en esa época, el Ayuntamiento estaba contratando a limpiadoras, y le hicieron una entrevista: "yo contando mis penas..., y justo llegué a casa, y me llamaron para trabajar ya [...]. Eso fue lo mejor que me ha pasado en mi vida..., aparte de mis hijos..., tener un trabajo". En 1981-1982, el ayuntamiento de la localidad decidió contratar a nueve personas, la mayoría mujeres, para la limpieza de algunos colegios del pueblo y de otros servicios municipales. Estos contratos se ofrecieron a personas de la localidad, con cargas familiares, sin empleo en el hogar familiar y en situación de vulnerabilidad social o riesgo de exclusión.

Su marido regresó a casa. Consiguió contratos eventuales, con empleos en los que era necesario trasladarse a otras provincias de España o a países europeos. Cuando trabajaba, los salarios eran altos, pero nunca consiguió contrato fijo; el contrato fijo era el de ella. Él trabajó hasta que tuvo un accidente de tráfico en otro país mientras estaba allí trabajando (1991-1992). Le correspondió una alta indemnización económica y la incapacidad absoluta, que le permitió cobrar una pensión.

A los años, la entrevistada comenzó a tener problemas de espalda y el Ayuntamiento la trasladó a tareas no físicas, fundamentalmente atendiendo el teléfono y la centralita. En total, estuvo trabajando 23 años. Lleva 13 años jubilada. 


\subsection{Análisis de los relatos de vida}

Después de exponer estos dos relatos, queremos hacer una pequeña comparación entre ellos: épocas en las que se desarrollaron las trayectorias de vida, contextos que incidieron en ellas y apoyos sociales que recibieron estas personas (redes sociales primarias y secundarias).

En primer lugar, nos encontramos en dos épocas distintas. Una, en la España de los años ochenta, en el inicio de la construcción del Estado del bienestar, sus políticas sociales y desarrollo embrionario de los servicios sociales. La otra, con el modelo de Bienestar instaurado, con sus dificultades, pero con más de veinte años de recorrido. Ambas épocas son años de crisis económica, de destrucción de empleo y de altas tasas de desempleo.

Según datos de la Encuesta de Población Activa (cit. en Sevillano, 2016), en los años ochenta la destrucción del empleo en Euskadi fue mayor y más prolongada en el tiempo que la crisis iniciada en 2008. Pero si prestamos atención a las tasas de temporalidad de los contratos, nos damos cuenta de que, al quedarse $A$. sin empleo, se encontró con un mercado de trabajo más precarizado que el que vivió $M$. Para el primero, fue más difícil encontrar empleo estable, lo que llevó a que fuera aumentando su situación de precariedad. A., tras renunciar a su último contrato en la administración pública, accedió a un contrato municipal de seis meses en fomento de empleo, distinto a la oportunidad que tuvo M. de conseguir un contrato laboral fijo, que mantuvo hasta su jubilación. Fueron años diferentes, en los que las políticas económicas abogaban más por la contratación pública de lo que se hace en la actualidad con las políticas de reducción del gasto público y subcontratación de muchos de sus servicios.

En segundo lugar, la red social de $M$. era más densa que la de A. Vivía en un barrio con un fuerte tejido asociativo y en el que las relaciones sociales eran menos individualizadas que las de las sociedades actuales. M. tuvo más oportunidades de hacer frente a su situación por el apoyo social del que disponía. A. había perdido a sus padres, había roto con su hermana, dejó de relacionarse con sus amigos y entró en zona de vulnerabilidad en una época con menos densidad de vínculos sociales.

En resumen, son tiempos distintos, aunque ambos de crisis económica. Uno, en el que el mercado de trabajo se fragmenta cada vez más y genera empleos precarios, y en el que la precariedad relacional también crece. Otro, con altas tasas de desempleo, pero en el que las recetas neoliberales todavía no se habían extendido en lo referente al gasto público, la contratación pública y los tipos de contratación; años, también, en los que los valores individualistas solamente comenzaban a imponerse. 


\section{Bibliografía referenciada}

ARRIBA GONZÁLEZ DE BURANA, A. (2002): El concepto de exclusión social en política social, serie Documentos de Trabajo, n-0 02-01, Madrid, Consejo Superior de Investigaciones Científicas, Unidad de Políticas Comparadas.

BECK, U. (2017): La metamorfosis del mundo, Barcelona, Paidós.

BENEDICTO XVI (2015): Deus Caritas est [encíclica], Ciudad del Vaticano, Libreria Editrice Vaticana.

BERTAUX, D. (1997): Los relatos de vida. Perspectiva etnosociológica, Barcelona, Bellaterra.

CÁRITAS ESPAÑOLA (2017): "LXXIV Asamblea General de Cáritas Española. Declaración final”.

- (2010): Propuestas políticas de Cáritas, serie Cáritas Actúa, n- 18, Madrid, Cáritas Española [<http://www.caritas.es/imagesrepository/ CapitulosPublicaciones/5514/boletin\%20 Actua18\%20web.pdf $>$ ].

CARRASCO, C. (2001): "La sostenibilidad de la vida humana: ¿un asunto de mujeres?", Mientras Tanto, no 82 , págs. 43-70.

CONSEJO ECONÓMICO Y SOCIAL VASCO (2017): Memoria socioeconómica 2016. Comunidad Autónoma del País Vasco / Memoria Sozioekonomikoa 2016. Euskal Autonomia Erkidegoa. Laburpena eta gogoetak, Bilbao, Consejo Económico y Social Vasco.

EUSTAT (2017): "La tasa de paro baja al 11\% y la población ocupada aumenta en 13.800 en el segundo trimestre de 2017 en la C.A. de Euskadi” [nota de prensa], 21-7-17, Vitoria-Gasteiz, EustatInstituto Vasco de Estadística [/http://www. eustat.eus/estad/clave_4/coyunturas.html〉].

- (2016): Encuesta sobre la Conciliación de la Vida Laboral, Familiar y Personal / Lana, Familia eta Norberaren Bizitza Bateratzeari buruzko Inkesta, Vitoria-Gasteiz, Eustat-Instituto Vasco de Estadística.

GARCÍA ROCA, J. (1998): Exclusión social y contracultura de la solidaridad. Prácticas, discursos y narraciones, Madrid, HOAC.

GOBIERNO VASCO. DEPARTAMENTO DE EMPLEO Y POLÍTICAS SOCIALES (2016a): Encuesta de Condiciones de Trabajo, Vitoria-Gasteiz, Eusko JaurlaritzaGobierno Vasco.

GOBIERNO VASCO. DEPARTAMENTO DE EMPLEO Y POLÍTICAS SOCIALES (2016b): Encuesta de Pobreza y Desigualdades Sociales EPDS-2016. Comunidad Autónoma de Euskadi. Principales resultados = Pobreziari eta Gizarte-desberdintasunei buruzko Inkesta PGDI-2016. Euskal Autonomia Erkidegoa. Emaitza nagusiak, Vitoria-Gasteiz, Eusko Jaurlaritza-Gobierno Vasco [<http:// www.eustat.eus/elementos/eleo014100/ ti_Encuesta_de_pobreza_y_desigualdades_. sociales_EPDS-2016_Principales_resultados PDF_16MB/info014126_c.pdf)].

LANBIDE (2017): “Expedientes activos de RGI. Junio 2017", Eusko Jaurlaritza-Gobierno Vasco [«http:// www.lanbide.euskadi.eus/y94-estadist/es/ contenidos/estadistica/rgi_beneficiarios_ territ_2017/es_def/adjuntos/o6-Junio/ rgithcae.shtml>].

- (2016): Estudio de incorporación a la vida laboral. Promoción universitaria de 2012. Conjunto de titulaciones. Universidad del País Vasco / Euskal Herriko Unibertsitatea, Vitoria-Gasteiz, Lanbide.

MILLS, C. W. (1959): La imaginación sociológica, Fondo de Cultura Económica.

PABLO VI (1965): Gaudium et Spes [constitución pastoral]. 
PÉREZ MOYA, C. (2016): "La pobreza y el nuevo contexto de la exclusión social”, Publicaciones Didácticas, nํ- 77, págs. 479-483.

PÉREZ-OROZCO, A.; y DEL RÍO, S. (s/d): "Una visión feminista de la precariedad desde los cuidados".

SEVILLANO, J. (2016): “Datos desde 1976, del número de: afiliados a la Seguridad Social, parados y tasa de paro y ocupados" [<https://javiersevillano. es/EPADatos.htm>].

ZUBERO, I. (2006): “Las nuevas relaciones entre empleo e inclusión: flexibilización del trabajo y precarización vital”, Documentación Social, nํㅜ 143, págs. 11-30. 